\title{
Suscetibilidade a antifúngicos de variedades de Cryptococcus neoformans isoladas de pacientes em hospital universitário
}

\author{
Susceptibility to antifungal agents among Cryptococcus neoformans \\ varieties isolated from patients at a university hospital
}

\author{
Paulo Roberto da Silva1, Rosiley Aparecida de Souza Rabelo', \\ Ana Paula Sarreta Terra ${ }^{2}$ e David Nascimento Silva Teixeira ${ }^{2}$
}

\begin{abstract}
RESUMO
Este trabalho identificou variedades de Cryptococcus neoformans e avaliou a suscetibilidade a antifúngicos pelo protocolo M27-A2 do National Committee for Clinical Laboratory Standards em isolados de 35 pacientes do Hospital Escola da Universidade Federal do Triângulo Mineiro. A variedade gatti foi identificada em $11.4 \%\left(\mathrm{n}^{0}=4\right)$ dos casos. A concentração inibitória mínima ( $\left.\mu \mathrm{g} / \mathrm{ml}\right)$ dos isolados de Cryptococcus neoformans neoformans variou de 0,062 - 2,000 (anfotericina B), 0,250 - 8,000 (fluconazol), 0,062 - 1,000 (itraconazol) e 0,125 - 1,000 (cetoconazol). A variedade gattii apresentou concentração inibitória mínima de 0,125 - 2,000 (anfotericina B), 0,250 - 16,00 (fluconazol), 0,062 - 1,000 (itraconazol) e 0,125 - 4,000 (cetoconazol). Detectaram-se 2 isolados resistentes ao itraconazol e 2 a anfotericina B ( 1 isolado de cada variedade por antifúngico). Os dados mostram a importância da determinação da variedade e da concentração inibitória mínima de isolados de Cryptococcus neoformans para monitorar o desenvolvimento de resistência e possibilitar uma terapia mais adequada na criptococose.
\end{abstract}

Palavras-chaves: Cryptococcus neoformans. Cryptococcus gatti. Suscetibilidade. Agentes antifúngicos. Criptococoses.

\begin{abstract}
This study identified Cryptococcus neoformans varieties isolated from 35 patients at teaching hospital of the Federal University of the Triângulo Mineiro and evaluated the susceptibility to antifungal agents among these samples using the protocol M27-A2 from the National Committee for Clinical Laboratory Standards. The gattii variety was identified in $11.4 \%$ of the cases $(\mathrm{n}=4)$. The minimum inhibitory concentration $(\mu \mathrm{g} / \mathrm{ml})$ of Cryptococcus neoformans neoformans isolates ranged from 0.062 to 2.000 (amphotericin B), 0.250 to 8.000 (fluconazole), 0.062 to 1.000 (itraconazole) and 0.125 to 1.000 (ketoconazole). The gattii variety presented a minimum inhibitory concentration range of 0.125 to 2.000 (amphotericin B), 0.250 to 16.00 (fluconazole), 0.062 to 1.000 (itraconazole) and 0.125 to 4.000 (ketoconazole). Two isolates resistant to itraconazole and two resistant to amphotericin B (one isolate of each variety per antifungal agent) were found. These data show the importance of determining the variety and minimum inhibitory concentration of Cryptococcus neoformans isolates, in order to monitor resistance development and enable better treatment for cryptococcosis.
\end{abstract}

Key-words: Cryptococcus neoformans. Cryptococcus gattii. Susceptibility. Antifungal agents. Cryptococcosis.

o Cryptococcus neoformans é uma levedura ovalada com uma cápsula rica em polissacarídeos sendo normalmente encontrado associado a fezes de aves (Columba lívia) ou árvores (Eucalipitus), e cuja maior proporção de indivíduos afetados são aqueles portadores do vírus da imunodeficiência humana (HIV $)^{13}$. De acordo com a Coordenação Nacional de DST/AIDS do Ministério da Saúde, no período de 1980 a 2002, 6\% das infecções oportunistas encontradas em pacientes HIV positivos foram causadas pelo Cryptococcus neoformans ${ }^{12}$. Considerando as infecções fúngicas oportunistas que acometem pacientes portadores de HIV no mundo, a criptococose é a segunda mais frequiente ${ }^{13}$.

Segundo diversos estudos ${ }^{6}{ }^{11}$ o Cryptococcus neoformans pode ser classificado em duas variedades de acordo com os seus antígenos polissacarídeos capsulares, o Cryptococcus neoformans variedade neoformans (sorotipos A, D e AD) e 0

1. Disciplina de Microbiologia, Departamento de Ciências Biológicas, Universidade Federal do Triângulo Mineiro, Uberaba, M.G. 2. Disciplina de Laboratório Clínico, Departamento de Clínica Médica, Universidade Federal do Triângulo Mineiro, Uberaba, M.G.

Apoio financeiro: FAPEMIG, CNPq, FUNEPU, UFTM.

Endereço para correspondência: Prof. David Nascimento Silva Teixeira. Disc. de Laboratório Clínico/Dept ${ }^{\circ}$ de Clínica Médica/UFTM. Av. Frei Paulino 30, $38025-180$ Uberaba MG.

Tel: 5534 3318-5542, Fax: 5534 3336-7015

e-mail: silvateixeira@mednet.com.br

Recebido para publicação em: 04/06/2007

Aceito em: 10/03/2008 
Cryptococcus neoformans variedade gattii (sorotipos B e C). A variedade neoformans é cosmopolita e tem como principal ambiente as fezes de pombos e outras aves ${ }^{10}$. Já a variedade gattii é encontrada preferencialmente no meio campestre, em regiões tropicais e semitropicais, sendo o primeiro isolamento descrito em 1990 a partir de uma árvore (Eucalyptus calmadulensis) ${ }^{7}$. Em indivíduos imunocomprometidos, a principal variedade encontrada como responsável pela criptococose tem sido a neoformans, enquanto que nos imunocompetentes ocorre a predominância da variedade gattii ${ }^{25}$. Além disto, existem relatos de uma maior dificuldade e menor resposta terapêutica ao tratamento com antifúngicos nas infecções pela variedade gattii $^{1825}$. 0 aparecimento de infecções fúngicas reincidentes e a resistência de determinadas leveduras à terapia usual tem estimulado o uso de testes de suscetibilidade in vitro para escolha e acompanhamento da quimioterapia antifúngica mais adequada a cada caso $^{25}$.

Existem muito poucos trabalhos sobre identificação das variedades de Cryptococcus neoformans isoladas na região do Triângulo Mineiro ${ }^{15}$. Diante disto, nosso trabalho teve como objetivo avaliar a suscetibilidade in vitro a quatro antifúngicos (anfotericina B, fluconazol, cetoconazol e itraconazol) segundo o protocolo M27-A 2002 do Clinical and Laboratory Standards Institute (CLSI), antigo National Committee for Clinical Laboratory Standards (NCCLS) ${ }^{16}$, de variedades de Cryptococcus neoformans isoladas de pacientes com criptococose, atendidos no Hospital Escola da Universidade Federal do Triângulo Mineiro (HE-UFTM).

\section{MATERIAL E MÉTODOS}

Amostras biológicas de paciente com criptococose. Neste trabalho, oram estudadas 35 amostras de Cryptococcus neoformans isoladas pelo Serviço de Patologia Clínica da UFTM a partir do líquido cefalorraquidiano (LCR), raspado de lesão (pele), lavado brônquio-alveolar e sangue venoso periférico de pacientes durante o diagnóstico de criptococose. Cepas controle cedidas pela Universidade Federal de Goiás (UFG), previamente identificadas como CBS 132, (sorotipo A, variedade neoformans) e ICB 565 (sorotipo B, variedade gattii) também foram avaliadas.

Dados clínicos: Os dados dos pacientes tais como idade, sexo, procedência e sorologia para HIV foram obtidos no Serviço de Arquivo Médico (SAME) da UFTM e organizados em bancos de dados. A maioria dos pacientes acometidos de criptococose, $85,7 \%\left(n^{0}=30\right)$, foi do sexo masculino, sendo $42,8 \%$ $\left(\mathrm{n}^{\mathrm{0}}=15\right)$ na faixa etária entre trinta e um a quarenta anos. Com relação à sorologia para HIV, 85,7\% $\left(n^{0}=30\right)$ dos pacientes tinham sorologia positiva. Em 11,4\% $\left(n^{-0}=4\right)$ dos casos, encontramos outras causas de imunossupressão identificadas como lipossarcoma $\left(\mathrm{n}^{\circ}=1\right)$, lupus $\left(\mathrm{n}^{\circ}=1\right)$, transplante renal $\left(n^{0}=1\right)$ diabetes $\left(n^{\underline{0}}=1\right)$. Apenas um paciente não apresentou fatores predisponentes para a criptococose. Com relação ao tratamento e evolução, 88,6\% (n-0 = 31) dos pacientes utilizaram anfotericina $B$, com doses variando de 20 a $30 \mathrm{mg} / \mathrm{kg}$, sendo que
$68 \%$ destes $\left(n^{0}=21\right)$ também fizeram uso de fluconazol. 0 paciente em que foi isolado o Cryptococcus neoformans de raspado lesão de couro cabeludo foi tratado somente com o itraconazol e três pacientes foram tratados somente com fluconazol. Dos 35 pacientes diagnosticados com criptococose, 26 (74,2\%) foram a óbito. Em relação às formas de manifestação da criptococose, dos 35 pacientes estudados, $30(85,7 \%)$ foram diagnosticados como neurocriptococose, dois pacientes apresentaram criptococose pulmonar, um foi acometido por criptococose cutânea e em dois casos o Cryptococcus neoformans foi detectado somente por hemocultura. 0 presente trabalho foi aprovado pelo Comitê de Ética em Pesquisa da UFTM com o número de protocolo 677.

Identificação das variedades de Cryptococcus neoformans isoladas. Inicialmente, foi identificada a presença de fungo encapsulado nas amostras biológicas por exame direto em microscopia de luz na presença de tinta da nanquim. Após a identificação as amostras foram cultivadas em ágar Sabouraud dextrose (DIFCO-USA), em estufa a $35 \pm 1^{\circ} \mathrm{C}$. Colônias sugestivas de Cryptococcus neoformans foram repicadas em meio ágar niger e meio de Christensen, para avaliar-se respectivamente a atividade de fenoxidase e uréase, além da realização do teste de assimilação de açúcar inositol. Quando os resultados de todas estas provas eram positivos identificava-se a espécie Cryptococcus neoformans ${ }^{426}$. As variedades de Cryptococcus neoformans foram identificadas pelo meio L-canavanina-glicina-azul de bromotimol (CGB) ${ }^{11}$.

Teste de suscetibilidade a antifúngicos. Foi realizado o teste de microdiluição em caldo utilizando método proposto pelo CLSI (Clinical and Laboratory Standards Institute), antigo NCCLS (National Committee for Clinical Laboratory Standards) segundo protocolo M-27-A2 ${ }^{16}$ para determinar a concentração inibitória mínima (CIM), visando avaliar o perfil de suscetibilidade das variedades de Cryptococcus neoformans aos derivados azólicos: itraconazol, cetoconazol, e fluconazol (Genix-Índia) e ao poliênico anfotericina B (Cristália-Brasil). Todos os testes foram realizados em duplicata.

Controle de qualidade. Uma cepa controle de Candida parapsilosis, (ATCC 22019) com CIMs pré-estabelecidas foi testada em cada experimento realizado para a validação dos resultados obtidos para as amostras de Cryptococcus neoformans. Também foram utilizadas duas cepas controle de Cryptococcus neoformans identificadas como CBS 132, (sorotipo A, variedade neoformans) e ICB 565 (sorotipo B, variedade gattii). Todas estas cepas foram cedidas pela Universidade Federal de Goiás (UFG) e apresentaram resultados equivalentes aos obtidos pelo nosso estudo quando avaliadas previamente frente aos antifúngicos dos fabricantes que os patentearam. Como o protocolo M27-A2 ${ }^{16}$ do NCCLS não propôs valores limites de suscetibilidade para o Cryptococcus neoformans, foram considerados resistentes os isolados que apresentaram CIM $\geq 64 \mathrm{~g} / \mathrm{ml}$, para fluconazol, $\mathrm{CIM} \geq 1 \mathrm{~g} / \mathrm{ml}$ para 0 itraconazol e CIM $\geq 2 \mu \mathrm{g} / \mathrm{mL}$ para a anfotericina $\mathrm{B}$ conforme sugerido previamente por estudos de outros autores ${ }^{17} 192224$. Os dados foram apresentados em CIM $_{50}$ e $\mathrm{CIM}_{90}$, que correspondem à concentração de cada antifúngico capaz de inibir $50 \%$ ou $90 \%$ dos isolados, respectivamente. 


\section{RESULTADOS}

Suscetibilidade dos isolados aos antifúngicos. $\mathrm{Na}$ Tabela 1, são apresentados a CIM $_{50}$, CIM $_{90}$ e a variação da CIM para os quatros antifúngicos estudados frente às variedades de Cryptococcus neoformans isoladas. Com relação ao cetoconazol, todos os isolados da variedade neoformans foram considerados susceptíveis in vitro este antifúngico. Os isolados da variedade gattii também foram susceptíveis à ação do cetoconazol, porém apresentando valores de $\mathrm{CIM}_{50}(0,5 \mu \mathrm{g} / \mathrm{mL})$ e CIM $\mathrm{CI}_{90}(4 \mu \mathrm{g} / \mathrm{mL})$ mais elevados do que os encontrados para a variedade neoformans. Os isolados da variedade neoformans foram susceptíveis ao fluconazol e dentre as amostras de variedade gattii, um dos isolados apresentou um valor mais elevado de CIM $(16 \mu \mathrm{g} / \mathrm{mL})$, porém ainda considerado susceptível a este antifúngico. As variedades gatti e neoformans apresentaram perfis equivalentes de suscetibilidade ao itraconazol, com a maioria dos isolados susceptivel a este antifúngico, porém foram encontradas duas amostras resistentes com CIM de $1 \mu \mathrm{g} / \mathrm{mL}$, sendo uma de cada variedade. Em relação à anfotericina $\mathrm{B}$, foram detectados dois isolados resistentes com CIM igual a $2 \mu \mathrm{g} / \mathrm{mL}$, sendo um da variedade neoformans e outro da variedade gattii. Os resultados de CIM para a cepa contrôle de Candida parapsilosis, (ATCC 22019) utilizado em nossos experimentos foram equivalentes aos valores de referência descritos pelo NCCLS.

Tabela 1 - Suscetibilidade in vitro a antifúngicos de variedades de Cryptococcus neoformans isoladas de pacientes atendidos no Hospital Escola da Universidade Federal do Triângulo Mineiro.

\begin{tabular}{|c|c|c|c|c|}
\hline \multicolumn{5}{|l|}{ Agente antifúngico } \\
\hline \multirow{2}{*}{$\begin{array}{l}\text { e variedade de } \\
\text { Cryptococcus neoformans }\end{array}$} & \multirow{2}{*}{$\begin{array}{c}\text { Isolados } \\
\left(\mathrm{n}^{0}\right)\end{array}$} & \multicolumn{3}{|c|}{ CIM (mg/ml) } \\
\hline & & variação & $\mathrm{CIM}_{50}$ & $\mathrm{CIM}_{90}$ \\
\hline \multicolumn{5}{|l|}{ Anfotericina B } \\
\hline variedade neoformans & 31 & $0,062-2,000$ & 0,250 & 1,000 \\
\hline variedade gattii & 4 & $0,125-2,000$ & 0,125 & 2,000 \\
\hline \multicolumn{5}{|l|}{ Fluconazol } \\
\hline variedade neoformans & 31 & $0,250-8,000$ & 4,000 & 4,000 \\
\hline variedade gattii & 4 & $0,250-16,00$ & 8,000 & 16,00 \\
\hline \multicolumn{5}{|l|}{ Itraconazol } \\
\hline variedade neoformans & 31 & $0,062-1,000$ & 0,125 & 0,500 \\
\hline variedade gattii & 4 & $0,062-1,000$ & 0,062 & 1,000 \\
\hline \multicolumn{5}{|l|}{ Cetoconazol } \\
\hline variedade neoformans & 31 & $0,125-1,000$ & 0,250 & 0,500 \\
\hline variedade gattii & 4 & $0,125-4,000$ & 0,500 & 4,000 \\
\hline
\end{tabular}

$\mathrm{CIM}_{50}$ e $\mathrm{CIM}_{90}$ : representam a concentração de antifúngico capaz de inibir, respectivamente, 50 e $90 \%$ dos isolados de Cryptococcus neoformans.

Valores máximos de Concentração Inibitória Mínima quanto ao local de isolamento. Quanto ao local de isolamento podemos observar que, em geral, as amostras de Cryptococcus neoformas obtidas do líquor apresentaram valores mais elevados de CIM para todos os antifúngicos testados quando comparados com os isolados de sangue, pele e lavado brônquio-alveolar (Tabela 2). A exceção ocorreu para o caso específico da suscetibilidade in vitro dos isolados do lavado brônquio-alveolar frente ao fluconazol. Neste caso, as amostras apresentaram valores de CIM elevados, equivalente àqueles encontrados para o líquor.
Tabela 2 - Variação da Concentração Inibitória Mínima quanto ao local de isolamento das amostras de Cryptococcus neoformans.

\begin{tabular}{|c|c|c|c|c|}
\hline \multirow[b]{2}{*}{ Agente antifúngico } & \multicolumn{4}{|c|}{ Variação da CIM $(\mathrm{mg} / \mathrm{ml}) /$} \\
\hline & $\begin{array}{c}\text { líquor } \\
\left(n^{0}=30\right)\end{array}$ & $\begin{array}{l}\text { sangue } \\
\left(\mathrm{n}^{\mathrm{o}}=2\right)\end{array}$ & $\begin{array}{l}\text { lavado brônquio-alveolar } \\
\qquad\left(n^{0}=2\right)\end{array}$ & $\begin{array}{c}\text { pele } \\
\left(\mathrm{n}^{\mathrm{o}}=1\right)\end{array}$ \\
\hline Anfotericina B & $0,062-2,000$ & $0,250-0,250$ & $0,125-0,125$ & 0,062 \\
\hline Fluconazol & $0,250-16,00$ & $2,000-2,000$ & $2,000-8,000$ & 2,00 \\
\hline Itraconazol & $0,062-1,000$ & $0,125-0,125$ & $0,062-0,062$ & 0,062 \\
\hline Cetoconazol & $0,250-4,000$ & $0,250-0,250$ & $0,062-0,500$ & 0,062 \\
\hline
\end{tabular}

CIM: representa a concentração de antifúngico capaz de inibir in vitro o crescimento do respectivo isolado de Cryptococcus neoformans.

\section{DISCUSSÃO}

No presente trabalho, a maioria dos casos diagnosticados de criptococose no HE-UFTM apresentou a variedade neoformans como principal agente etiológico (Tabela 1). Neste aspecto, nossos dados são similares aos estudos feitos em outras regiões que apontam esta variedade como a principal responsável por casos de criptococose em pacientes com a síndrome da imunodeficiência adquirida (SIDA) ${ }^{1325}$.

A maioria dos estudos encontrados na literatura mostra que isolados de Cryptococcus neoformans apresentam suscetibilidade in vitro a diferentes antifúngicos ${ }^{24}$. A resistência de Cryptococcus neoformans aos antifúngicos in vitro é fenômeno pouco freqüente, embora existam alguns relatos ${ }^{20}{ }^{24}$. Neste trabalho, detectamos a presença de isolados clínicos resistentes ao itraconazol e a anfotericina B na região do Triângulo Mineiro. Com relação ao fluconazol, não foram detectados Cryptococcus neoformans resistentes a este antifúngico, embora um isolado da variedade gattii tenha apresentado uma CIM relativamente elevada $(16 \mathrm{~g} / \mathrm{mL})$. A terapia de longa duração com o fluconazol na criptococose tem sido descrita como um dos fatores associados ao desenvolvimento de cepas resistentes a este antifúngico ${ }^{5131721}$. Dentro deste contexto, o itraconazol tem sido apontado como uma alternativa interessante ao fluconazol, devido à alta suscetibilidade in vitro de isolados de Cryptococcus neoformans a este antifúngico descrita em estudos anteriores ${ }^{9}$. Diferentemente de outros trabalhos da literatura ${ }^{8928}$ encontramos em nosso estudo dois isolados clínicos resistentes ao itraconazol com CIM de $1 \mathrm{~g} / \mathrm{mL}$ (um da variedade neoformans e outro da gattii). Estes resultados sugerem que o uso alternativo do itraconazol ao fluconazol também deve ser avaliado com cautela, pois apesar da atividade in vitro do fluconazol ser baixa em alguns estudos, acredita-se que in vivo este antifúngico possa atingir níveis plasmáticos mais elevados que 0 itraconazol ${ }^{14}$.

A anfotericina B tem sido freqüentemente utilizada no tratamento inicial da criptococose ${ }^{13}$, sendo que a detecção de cepas de Cryptococcus neoformans resistentes a este antifúngico é infrequiente. No entanto, encontramos dois isolados resistentes a anfotericina B em nosso estudo (um da variedade neoformans e outro da variedade gattii). Nossos dados também mostram que a variedade gatti apresenta uma tendência a exibir CIMs um pouco mais elevados do que a variedade neoformans, reforçando, em parte, observações anteriores de que esta variedade é de terapia mais difícil e prolongada se comparada a variedade neoforman ${ }^{2527}$. 
Os resultados obtidos também mostraram que os Cryptococcus neoformans isolados de liquor apresentaram CIMs mais elevados do que as amostras biológicas coletadas à partir de outros locais. A exceção ocorreu para os isolados de lavado brônquio-alveolar quando testados frente a fluconazol que apresentaram, neste caso, valores de CIMs equivalentes àqueles encontrados no liquor. 0 aparecimento de cepas de Cryptococcus neoformans resistentes está geralmente associado com infecções crônicas encontradas em indivíduos imunocomprometidos e com neurocriptococose, sendo mais facilmente encontradas, nestes casos, cepas resistentes ou com CIMs elevados nas amostras de líquor rotineiramente utilizadas para o diagnóstico laboratorial destes pacientes ${ }^{1323}$. Embora tenham sido isoladas somente duas cepas no lavado brônquio-alveolar, estas apresentaram CIMs mais elevados para fluconazol, devido provavelmente ao seu uso constante por pacientes imunocomprometidos por SIDA, podendo o fungo inicialmente causar uma infecção pulmonar sintomática ou não e posteriormente disseminar-se para as meninges e causar neurocriptococose.

Em conclusão, os dados obtidos mostram que a variedade neoformans foi a mais prevalente como causadora de criptococose em pacientes atendidos no Hospital Escola da UFTM. A maioria dos isolados de Cryptococcus neoformans apresentou um perfil de suscetibilidade in vitro, sendo encontrado apenas dois isolados não-suscetíveis, indicando que a resistência aos antifúngicos convencionais ainda não é um problema significativo. Todavia, a realização de estudos que detectem a variedade e a CIM de isolados de Cryptococcus neoformans é importante para se monitorar 0 aparecimento de novas cepas resistentes.

\section{AGRADECIMENTOS}

Os autores agradecem a $\operatorname{Prof}^{a}$ Orionalda de Fátima L. Fernandes e a Prof ${ }^{a}$ Lúcia K.H. Souza da Universidade Federal de Goiás (UFG) que gentilmente cederam as cepas controle de Cryptococcus neoformans e Candida parapsilosis utilizadas neste estudo.

\section{REFERÊNCIAS}

1. Baker DJ, Reboli AC. Images in clinical medicine: Cutaneous cryptococcosis. New England Journal of Medicine 336: 998, 1997.

2. Calhoun DL, Roberts GD, Galgiani JN, Bennett JE, Feingold DS, Jorgensen J, Kobayashi GS, Shadomy S. Results of survey of antifungal susceptibility tests in the United States and interlaboratory comparison of broth dilution testing of flucytosine and amphotericin. Bangladesh Journal of Clinical Microbiology 23: 298-301, 1986.

3. Chen S, Sorrell T, Nimmo G, Speed B, Currie B, EllisD, Marriott D, Pfeiffer T, Parr D, Byth K. Australasian Cryptococcal Study Group Epidemiology and Host- and variety-dependent characteristics of infection due to Cryptococcus neoformans in Australia and NewZealand. Clinical Infectious Diseases 31: 499-508, 2000.

4. Christensen WB. Urea decomposition as a means of differentiating Proteus and Paracolon cultures from each other and from salmonella and shigella types. Journal of Bacteriology 52: 461-466, 1946.

5. Colombo AL, Barchiesi F, Mcgough DA, Rinald MG. Comparison of Etest and National Committee for Clinical Laboratory Standards broth macrodilution method for azole antifungal susceptibility testing. Journal of Clinical Microbiology 33: 535-540, 1995.

6. Diaz MR, Boekhout T, Theelen B, Fell JW. Molecular sequence analyses of the intergenic spacer (IGS) associated with rDNA of the two varieties of the pathogenic yeast, Cryptococcus neoformans. Systematic and Applied Microbiology 23: 535-545, 2000

7. Ellis DH, Pfeiffer TJ. Ecology, life cycle, and infectious propagule of Cryptococcus neoformans. Lancet 336: 923-925, 1990.

8. Fernandes OFL, Passos XS, Souza LK, Miranda AT, Cerqueira CH, Silva MR. In vitro susceptibility characteristics of Cryptococcus neoformans varieties from AIDS patients in Goiania. Memórias do Instituto Osvaldo Cruz 98: 839-841, 2003.

9. Franzot SP, Hamdan JS, Currie BP, Casadevall A. Molecular epidemiology of Cryptococcus neoformans in Brazil and the United States: evidence for both local genetic differences and a global clonal population structure. Journal of Clinical Microbiology 35: 2243-2251, 1997.

10. Fromtling RA, Bulmer GS. Distribution of Cryptococcus neoformans in a natural site. Infection and Immunity 31: 560-563, 1981.

11. Kwon-Chung KJ, Polacheck I, Bennett JE. Improved diagnostic medium for separation of Cryptococcus neoformans var. neoformans (serotypes A and D) and Cryptococcus neoformans var gattii (serotypes B and C). Journal of Clinical Microbiology 15: 535-537, 1982.

12. Ministério da Saúde. Dados e Pesquisa em DST e Aids. Coordenação do programa nacional de DST/Aids. Boletim Epidemiológico.AIDS/Ministério da Saúde. Brasília, 2002.

13. Mitchell TG, Perfect JR. Cryptococcosis in the era of AIDS - 100 years after the discovery of Cryptococcus neoformans. Clinical Microbiology and Infection 8: 515-548, 1995.

14. Moraes EM, Prímola NS, Hamdan JS Antifungal susceptibility of clinical and environmental isolates of Cryptococcus neoformans to four antifungal drugs determined by two techniques. Mycoses 46:164-168, 2003.

15. Moreira TA, Ferreira MS, Ribas RM, Borges AS. Cryptococosis: clinical epidemiologycal laboratorial study and fungi varieties in 96 patients. Revista da Sociedade Brasileira de Medicina Tropical 39: 255-258, 2006.

16. National Committee for Clinical Laboratory Standards (NCCLS). Reference method for broth dilution antifungal susceptibility testing of yeasts. Approved standard M27-A2. National Committee for Clinical Laboratory Standards, Wayne, PA. 2002

17. Nguyen $\mathrm{MH}, \mathrm{Yu} \mathrm{CY}$. In vitro comparative efficacy of voriconazole and itraconazole against fluconazole-susceptible and resistant Cryptococcus neoformans isolates. Antimicrobial Agents and Chemotherapy 42: 471-472, 1998.

18. Peachey PR, Gubbins PO, Martin RE. The association between cryptococcal variety and immunocompetent and immunocompromised hosts. Pharmacotherapy 18: 255-264, 1998.

19. Pedroso RS, Ferreira JC, Candido RC. In vitro susceptibility to antifungal agents of environmental Cryptococcus spp. isolated in the city of Ribeirão Preto, São Paulo, Brazil. Memórias do Instituto Osvaldo Cruz 101: 239-243, 2006.

20. Perkins A, Gomez-Lopez, A, Mellado Rodriguez-Tudela EJL, Cuenca-Estrella M. Rates of antifungal resistance among Spanish clinical isolates of Cryptococcus neoformans var. neoformans. Journal of Antimicrobial Chemotherapy 56: 1144-1147, 2005.

21. Pfaller MA, Boyken L, Hollis RJ, Messer AS, Tendolkar S, Diekema DJ. In vitro susceptibilities of clinical isolates of Candida species, Cryptococcus neoformans, and Aspergillus species to itraconazole: global survey of 9,359 isolates tested by clinical and laboratory standards institute broth microdilution methods. Journal of Clinical Microbiology 43: 3807-3810, 2005.

22. Rodriguez-Tudela JL, Berenguer Martiinez-Suarez JJ, Sanchez R. Comparison of a Spectrophotometric Microdilution Method with RPMI-2\% Glucose with the National Committee for Clinical Laboratory Standards Reference Macrodilution Method M27-P for in vitro Susceptibility Testing of Amphotericin B, Flucytosine, and Fluconazole against Candida albicans Antimicrobial Agents And Chemotherapy 40: 1998-2003, 1996.

23. Sar B, Monchy D, Vann M, Keo C, Sarthou JL, Buisson Y. Increasing in vitro resistance to fluconazole in Cryptococcus neoformans Cambodian isolates: April 2000 to March 2002. Journal of Antimicrobial Chemotherapy 54: 563-565, 2004. 
24. Souza LKH, Fernandes OFL, Kobayashi CCBA, Passos XS, Costa CR, Lemos JA, SouzaJúnior AH, Silva MRR. Antifungal susceptibilities of clinical and environmental isolates of Cryptococcus neoformans in Goiânia City, Goiás, Brazil. Revista do Instituto de Medicina Tropical de São Paulo 47: 253-256, 2005.

25. Speed B, Dunt D. Clinical and host differences between infections with the two varieties of Cryptococcus neoformans. Clinical Infectious Diseases 21: 28-34, 1995.
26. Staib F, Seibold M, Antweiler E, Fröhlich B, Weber S, Blisse A. The brown colour effect (BCE) of Cryptococcus neoformans in the diagnosis, control and epidemiology of Cryptococcus neoformans infections in AIDS patients. Zentralbl fur Bakteriologie, Mikrobiologie, und Hygiene Series A. 266: 167-177, 1987.

27. Trilles L, Fernández-Torres B, Lazéra MS, Wanke B, Guarro J. In vitro antifungal susceptibility of Cryptococcus gattii. Journal of Clinical Microbiology 42: 4815-4817, 2004 